\title{
AGB stars in the Magellanic Clouds and in other members of the Local Group
}

\author{
Maria-Rosa L. Cioni \\ European Southern Observatory, Karl-Schwarschild-Strasse 2, D-85748 \\ Garching bei Muenchen, Germany
}

\begin{abstract}
Results obtained in the Magellanic Clouds using the latest near-IR DENIS survey are briefly reviewed. This sets the stage for a similar study of AGB stars in other galaxies in the Local Group and, in particular, in NGC 6822 .
\end{abstract}

\section{Introduction}

There are two main advantages of studying asymptotic giant branch (AGB) stars in the galaxies of the Local Group (LG). First, that we are able, using the current instrumentation, to resolve their stellar content and second, that these stars can be considered to be approximately all at the same distance. Recent studies of AGB stars in the Magellanic Clouds allowed us to gain insight into the near-IR stellar content of the galaxies, their surface distribution and the ratio between carbon-rich (C-rich) and oxygen-rich (O-rich, M-type) AGB stars easily statistically distinguished in the colour-magnitude diagram (CMD, $J-K_{S}, K_{S}$ ). More recent near-IR observations of other galaxies in the LG have been analyzed to tell us similar information.

\section{Results on the Magellanic Clouds}

Among the latest near-IR instruments, DENIS and 2MASS have released a large amount of data on AGB stars in the Magellanic Clouds. In particular, in the DENIS catalogue towards the Magellanic Clouds (Cioni et al. 2000a) there are 32800 AGB stars in the Large Magellanic Cloud (LMC) and 7650 in the Small Magellanic Cloud (SMC), of which 7570 and 1640, respectively, are C-rich. Blanco et al. (1983) estimated about 11000 and 2900 C-rich stars in the LMC and SMC respectively. Allowing for about $10 \%$ misclassification among the M0-1 stars below the tip of the red giant branch (TRGB) which are missed by our selection criteria based on the near-IR CMD, we detect most of the AGB stars in both Galaxies. In fact we detect about the same number of $\mathrm{C}$ stars found by Kontizas et al. (2001) in the LMC (7750) and those 1707 found by Rebeirot et al. (1983) in the SMC.

AGB stars are easily distinguished in the $(I-J, I)$ CMD as the plume of objects above the TRGB redder than a given line that discriminates between the younger and older populations (Cioni et al. 2000b). These AGB stars distribute smoothly over the surface of the Clouds in contrast with the bar-like and patchy 

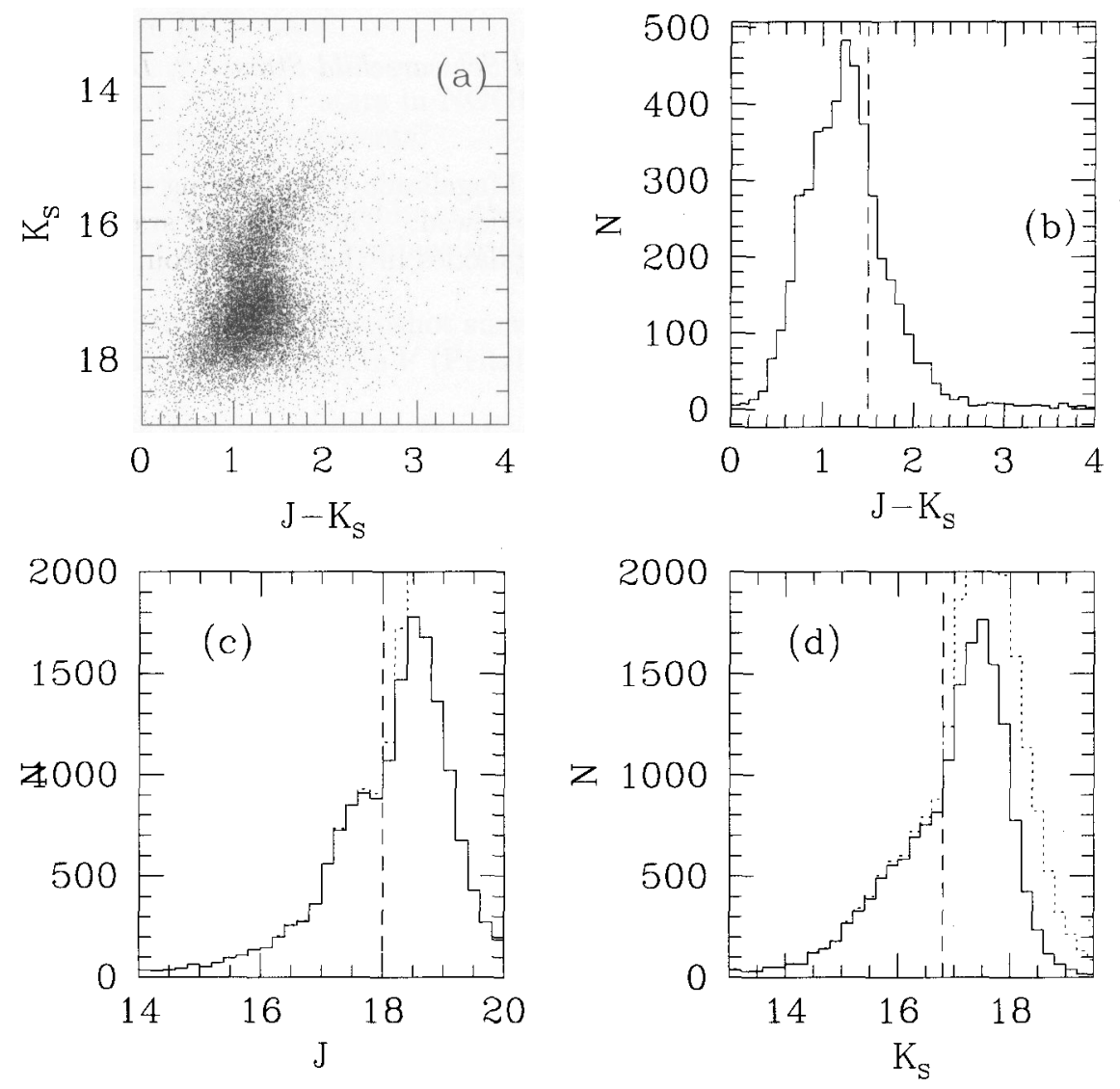

Figure 1. The colour-magnitude diagram and histograms in colour and $J$ and $K_{S}$ magnitudes for the sources observed towards NGC 6822 . (a) The $\left(J-K_{S}, K_{S}\right)$ diagram for all the sources detected within an area of $20^{\prime} \times 20^{\prime}$ centered on NGC 6822; (b) the histogram of the $J-K_{S}$ colour at about $0.3 \mathrm{mag}$ above the TRGB where the dashed line discriminates between O-rich and C-rich AGB stars; the histograms of $J$ (c) and $K_{S}$ (d) where the dashed line indicates the approximate location of the TRGB. 
distribution of the younger stars. In the $\left(J-K_{S}, K_{S}\right)$ CMD C-rich AGB stars occupy a red branch compared to the location of O-rich stars. The distribution of the ratio between $\mathrm{C}$-rich and O-rich (C/M ratio) AGB stars in the LMC outlines a ring-like structure of increasing values (Cioni \& Habing 2003), giving the first global evidence that there is a radial metallicity gradient in the LMC. The ratio is patchy and irregularly distributed in the SMC. By fitting the distribution of points $(\log (C / M)$ versus $[\mathrm{Fe} / \mathrm{H}])$ in other galaxies of the LG a metallicity spread of about 0.75 dex within each Cloud was derived. The larger uncertainty remains in the calibration and scatter of this relation.

\section{Results in other Local Group galaxies}

Based on the results obtained in the Magellanic Clouds, Habing and I started a near-IR campaign from the La Palma observatory to mosaic other galaxies of the LG in $I, J$ and $K_{S}$. The goal is to find, study and compare the AGB stellar population.

Fig. 1 shows: (a) the $\left(J-K_{S}, K_{S}\right)$ diagram for all the sources detected within an area of $20^{\prime} \times 20^{\prime}$ centered on NGC 6822 ; (b) the histogram of the $J-K_{S}$ colour at about $0.3 \mathrm{mag}$ above the TRGB where the dashed line discriminates between O-rich and C-rich AGB stars; and the histograms of $J$ (c) and $K_{S}$ (d), where the dashed line indicates the approximate location of the TRGB. In NGC 6822 we detect about $1339 \mathrm{C}$ stars, approximately 400 more than Letarte et al. (2002), and $2774 \mathrm{M}$ stars. The distribution of the whole AGB population is shown in Fig. 2 while Fig. 3 shows the distribution of the C/M ratio. Using the same relation discussed in Section 2, we derive that the gray scale and the contours span a range of $1.65 \mathrm{dex}$ in $[\mathrm{Fe} / \mathrm{H}]$. Contrary to Nowotny et al. (2003) we do detect regions with a different $\mathrm{C} / \mathrm{M}$ ratio. Note that in the $K_{S}$ band the differential reddening is negligible and that foreground stars have been removed. The striking difference between the two figures indicates the potential of the $\mathrm{C} / \mathrm{M}$ ratio to study the chemical history of galaxies.

Similar figures, though with less statistics, have been obtained for NGC 147 and NGC 185. Observations of Draco have just been reduced and unfortunately the observations of Leo A, Leo I and Leo II took place during variable sky conditions which considerably affect the quality of the resulting CMDs.

In the Southern Hemisphere the central regions of a few galaxies have been observed using SOFI at the NTT in the near-IR wave bands by Tolstoy back in 1998. The CMDs of DDO 210, Fornax, SagDIG and Pegasus show clear red giant branches reaching, in Fornax, the red clump. These galaxies are not rich in AGB stars, especially of $\mathrm{C}$ type, except Pegasus that shows a clump of objects with quite red $J-K_{S}$ colours. These relatively deep data are probably suitable to derive the metallicity and the age of the observed region (about $5^{\prime} \times 5^{\prime}$ ) from the slope and colour of the red giant branch as in Davidge (2003). New observations of these, and other, galaxies visible from the Southern Hemisphere took place at the end of July 2003 in collaboration with Reijkuba.

The wealth of near-IR data on galaxies in the Local Group other than the Magellanic Clouds will definitely benefit from the latest theoretical results by Marigo et al. (2003). These authors were able to successfully model the red tail of $\mathrm{C}$ stars, producing a synthetic $\left(K_{S}, J-K_{S}\right)$ diagram in very good agreement 

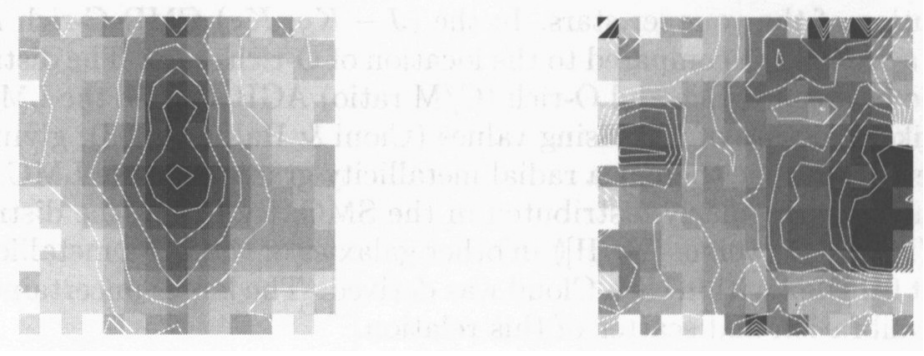

Figure 2. Spatial distribution of AGB stars (left) and C/M ratio (right) in NGC 6822 .

with the observational data. The key ingredient is to assume an opacity that in cool stars varies with the chemical composition in addition to the assumption of a given pulsation mode (the first overtone for $\mathrm{C}$ stars).

\section{Conclusions}

Large scale information on AGB stars in the Magellanic Clouds, NGC 6822, NGC 147 and NGC 185 have been discussed: their location in the near-IR CMD, their spatial distribution and that of the $\mathrm{C} / \mathrm{M}$ ratio. The latter is an important abundance indicator, especially for those systems too far away to measure abundances with other tracers. In the near future we will complete the reduction of complementary $I$-band measurements, continue observations of some targets not yet fully mosaiced, and carry on a monitoring program (in the $I$-band) that will provide us with an indication about the variability of the target stars. Ultimately we count on publishing homogeneous catalogues of AGB variables that will be useful to the whole community to perform stellar population studies in these nearby galaxies.

\section{References}

Blanco V.M., McCarthy M.F. 1983, AJ 88, 1442

Cioni M.-R.L., Habing H.J. 2003, A\&A 402, 133

Cioni M.-R.L., Habing H.J., Israel F.P. 2000b, A\&A 358, L9

Cioni M.-R.L., Loup C., Habing H.J., et al. 2000a, A\&A 144, 235

Davidge T.J. 2003, AJ 125, 3046

Kontizas E., Dapergolas A., Morgan D.H., et al. 2001, A\&A 369, 932

Letarte B., Demers S., Battinelli P., et al. 2002, AJ 123, 832

Marigo P., Girardi L., Chiosi C. 2003, A\&A 403225

Nowotny W., Kerschbaum F., Olofsson H., et al. 2003, A\&A 403, 93

Rebeirot E., Martin N., Prevot L., et al. 1983, A\&AS 53, 255 